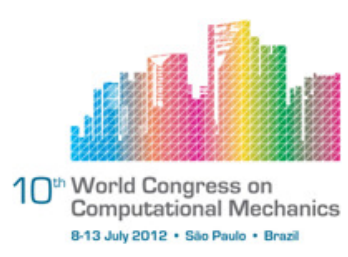

\title{
A COMPONENT MODE SYNTHESIS APPROACH FOR DYNAMIC ANALYSIS OF VISCOELASTICALLY DAMPED STRUCTURES
}

\author{
L. Rouleau ${ }^{1,2}$, J.-F. Deü ${ }^{1}$, A. Legay ${ }^{1}$, J.-F. Sigrist ${ }^{2}$, F. Le Lay ${ }^{2}$, P. Marin-Curtoud ${ }^{3}$ \\ ${ }^{1}$ Structural Mechanics and Coupled Systems Laboratory, Conservatoire National des Arts et \\ Métiers, 2 rue Conté, 75003 PARIS, France (lucie.rouleau@ cnam.fr) \\ ${ }^{2}$ DCNS Research, CESMAN, Indret, 44620 LA MONTAGNE, France \\ ${ }^{3}$ DGA Techniques Navales, BP 40915, 83050 TOULON Cedex, France
}

\begin{abstract}
The optimisation of structures with constrained viscoelastic layers is a major issue in the design of submarines, to ensure stealthiness performances. The first aim of this work is to develop a methodology to build reliable master curves of viscoelastic materials from dynamic mechanical analyzer measurements. The second aim is to propose reduction methods based on Craig-Bampton substructuring approach to solve the system composed of a mechanical structure with embedded damping material in the frequency domain. These strategies are applied to the response of a bidimensional viscoelastic sandwich ring with stiffeners.
\end{abstract}

Keywords: Viscoelasticity, Time-temperature superposition principle, Finite element models, Component mode synthesis.

\section{INTRODUCTION}

Passive damping control such as viscoelastic treatment is a widespread technique used to reduce vibration-induced noise in industrial applications. In naval shipbuilding, it has been used since the 1960 s to enhance submarine stealthiness performance. The viscoelastic material is generally embedded in the structure as a constrained layer since damping is proportional to shear stress. The need to optimize material and structural parameters of structures containing viscoelastic materials requires : a good representation of the viscoelastic behaviour, and reduction methods enabling the modelisation of large and complex systems. A first attempt has been made by the authors in [1] in order to optimize the placement of a viscoelastic patch on a bidimensional sandwich ring coupled to liquids. The first goal of the present work is to present the methodoly used to generate a reliable viscoelastic model from dynamical mechanical analyzer measurements. The second goal is to present reduction methods to calculate the frequency response of a sandwich structure with viscoelastic core, based on a component mode synthesis approach. 


\section{MASTER CURVES OF THE VISCOELASTIC MATERIAL}

The complex modulus approach is a common and convenient way of describing the frequency-dependence of the viscoelastic material properties:

$$
G^{*}(\omega)=|G(\omega)| \exp (\mathrm{i} \phi(\omega))
$$

A dynamic mechanical analyzer measures the amplitude of the complex modulus $|G|$ and the phase angle $\phi$ at different temperatures on a limited frequency range (Figures 1.a and 1.b). In order to know the viscoelastic behaviour of the material on a wider frequency range, the time-temperature superposition principle is generally applied. It stipulates that for a thermo-rheologically simple material, the modulus and the phase measured at a frequency $f$ and a temperature $T$ is equivalent to their values at a reduced frequency $f_{r}$ and a reference temperature $T_{0}[2]$ :

$$
\begin{array}{r}
\left|G\left(f_{r}, T_{0}\right)\right|=|G(f, T)| \\
\phi\left(f_{r}, T_{0}\right)=\phi(f, T)
\end{array}
$$

with $f_{r}=a_{T}\left(T, T_{0}\right) f$. The function $a_{T}\left(T, T_{0}\right)$ reflects the temperature dependence of relaxation time and corresponds to a horizontal shift of the isotherm measured at the temperature $T$, as shown on Figures 1.c and 1.d. The shift function $a_{T}\left(T, T_{0}\right)$ can be calculated according to the WLF equation [3]:

$$
\log \left(a_{T}\right)=\frac{-C_{1}\left(T-T_{0}\right)}{C_{2}+T-T_{0}}
$$

where $C_{1}$ and $C_{2}$ are coefficients depending on the material and the reference temperature $T_{0}$. The determination of the horizontal shift factors for each isotherm is based on a least square method minimizing the error between the shifted isotherms and a polynomial fit of the data. Figure 2 shows the temperature dependence of the function $a_{T}\left(T, T_{0}\right)$ used to obtain the master curves of Deltane 350, supplied by Paulstra Hutchinson, at a reference temperature of $T_{0}=12^{\circ} \mathrm{C}$, for measurements on the frequency range $0 \mathrm{~Hz}-258 \mathrm{~Hz}$ and on the temperature range $-50^{\circ} \mathrm{C}-+43^{\circ} \mathrm{C}$.

For many damping materials, the modulus depends weakly on the temperature, and no vertical shifting is necessary. However, for some materials, the horizontally shifted isotherms do not superimpose but fall onto paraller curves, and vertical shifting is required [4]. In this case Eq. 2 becomes:

$$
\left|G\left(f_{r}, T_{0}\right)\right|=b_{T}\left(T, T_{0}\right)|G(f, T)|
$$

where the function $b_{T}\left(T, T_{0}\right)$ reflects the temperature dependence of modulus and corresponds to a vertical shift of the isotherm measured at the temperature $T$, as shown on Figures 1.e and 1.f. For Deltane 350, vertical gaps are observed between the horizontally shifted isotherms (not presented here) which shows a need for vertical repositionning. The vertical shift factors are seaked such as the causality condition, inherent to the linear viscoelasticity assumption, is respected. Causality imposes relationships between the amplitude and the phase of the 
(a)

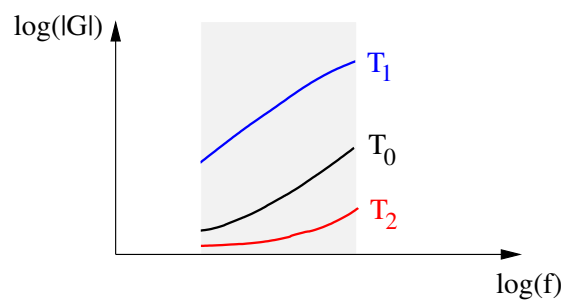

(c)

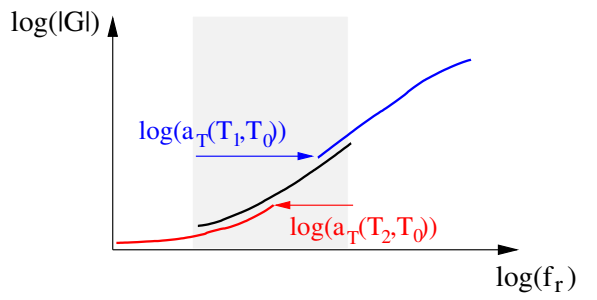

(e)

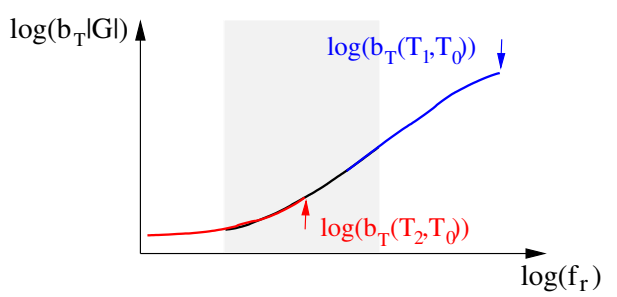

(b)

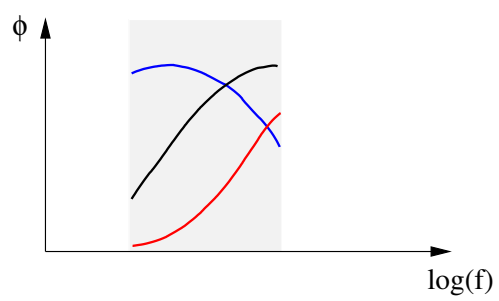

(d)

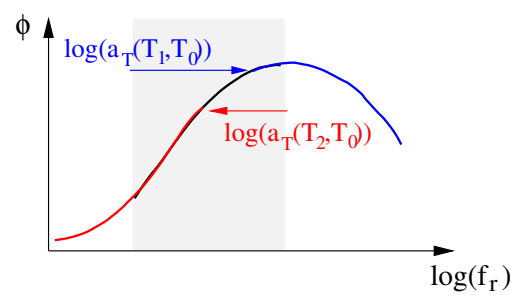

(f)

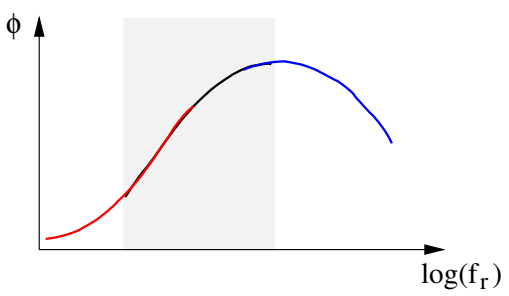

Figure 1. Time-temperature superposition principle. (a), (b) Isotherms of the complex modulus' amplitude and the phase angle on a limited frequency range with $T_{1}<T_{0}<T_{2}$. (c), (d) Horizontal shifting of the isotherms of complex modulus' amplitude and the phase angle with $T_{0}$ the reference temperature. (e), (f) Vertical shifting of the isotherms of complex modulus' amplitude with $T_{0}$ the reference temperature.

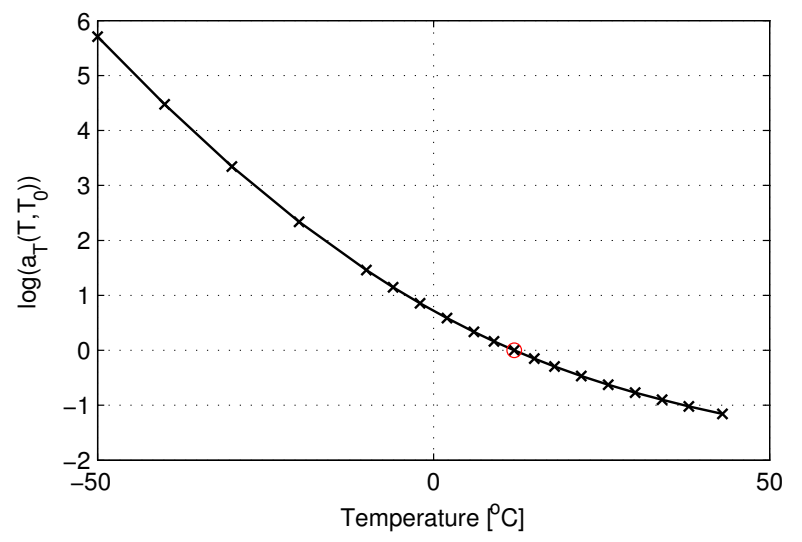

Figure 2. Temperature dependence of the function $a_{T}\left(T, T_{0}\right)$ used to obtain the master curves of the Deltane 350, at a reference temperature $T_{0}=12^{\circ} \mathrm{C}$. Crosses : temperature of measurements, circle : reference temperature. 

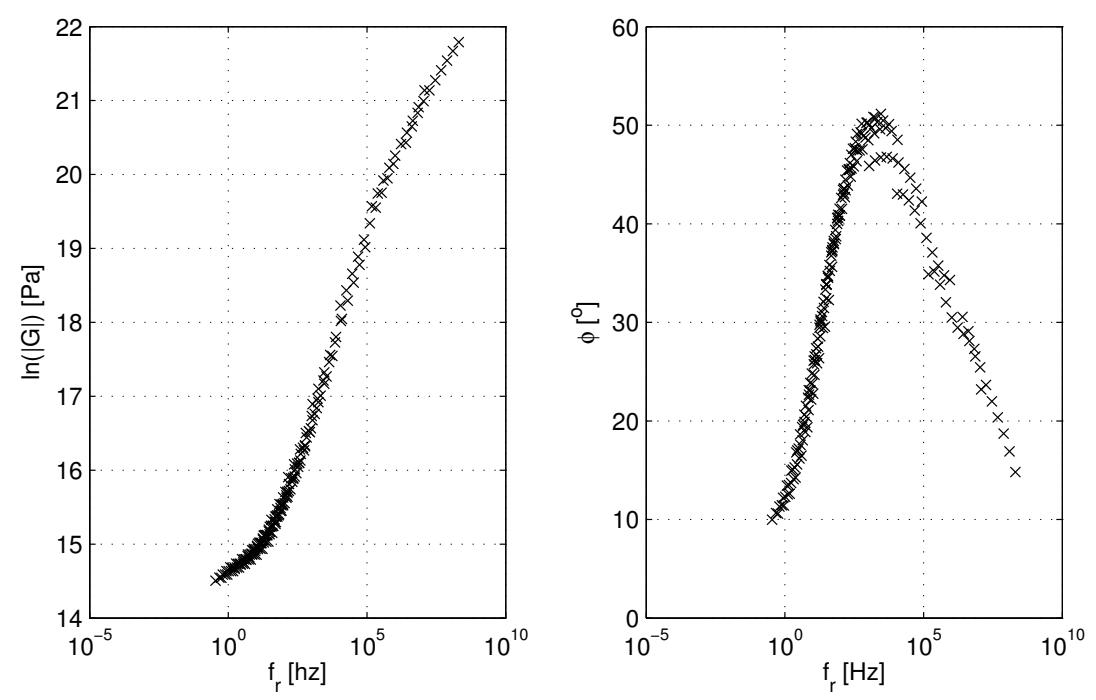

Figure 3. Master curves of Deltane 350 , at a reference temperature of $12^{\circ} \mathrm{C}$, after horizontal and vertical shifting.

complex modulus, namely the Kramers-Kronig relations:

$$
\begin{array}{r}
\ln (|G(\omega)|)_{K K}=\lim _{\omega \rightarrow+\infty} \ln (|G(\omega)|)_{K K}+\frac{2}{\pi} \int_{0}^{+\infty} \frac{u \phi(u)}{\omega^{2}-u^{2}} \mathrm{~d} u \\
\phi_{K K}(\omega)=\frac{2 \omega}{\pi} \int_{0}^{+\infty} \frac{\ln (|G(u)|)}{u^{2}-\omega^{2}} \mathrm{~d} u
\end{array}
$$

In [5], an approximation of those relations is used to determine the horizontal shift coefficients. However, Parot and Duperray [6] show that this approximation is not satisfactory, and propose instead a numerical method to evaluate the exact Kramers Kronig relations. This method, based on the integration of piecewise linear functions, allows the calculation of the complex modulus' amplitude from the phase angle, and vice versa. The causality index, introduced in [6], is a way of measuring the deviation from ideal causal data. It is defined as:

$$
I(\omega)=\frac{1}{2}\left|\frac{\ln \left(G_{K K}^{*}(\omega)\right)-\ln \left(G^{*}(\omega)\right)}{\ln \left(G_{K K}^{*}(\omega)\right)}\right|
$$

where the subscript ${ }_{K K}$ refers to the data computed from Eq.(6).

The closer to zero this index gets, the more causal the data are. In our work, a least square method minimizing the norm of this causality index is used to determine the optimal vertical shift factors. The master curves obtained at the end of the vertical repositionning of the isotherms are shown on Figure 3. The causality index is plotted as a function of frequency on Figure 4. The oscillations observed are due to the discontinuities of the master curves at the junction of isotherms. The norm of this index is reduced from 0.364 to 0.029 by introducing a vertical shifting. The calculated vertical shift coefficients are given on Figure 5. They appear to lie on a curve and to have a similar temperature dependence to that of the horizontal shift coefficients (Figure 2). This unexplained result requires more study and will be investigated further. 


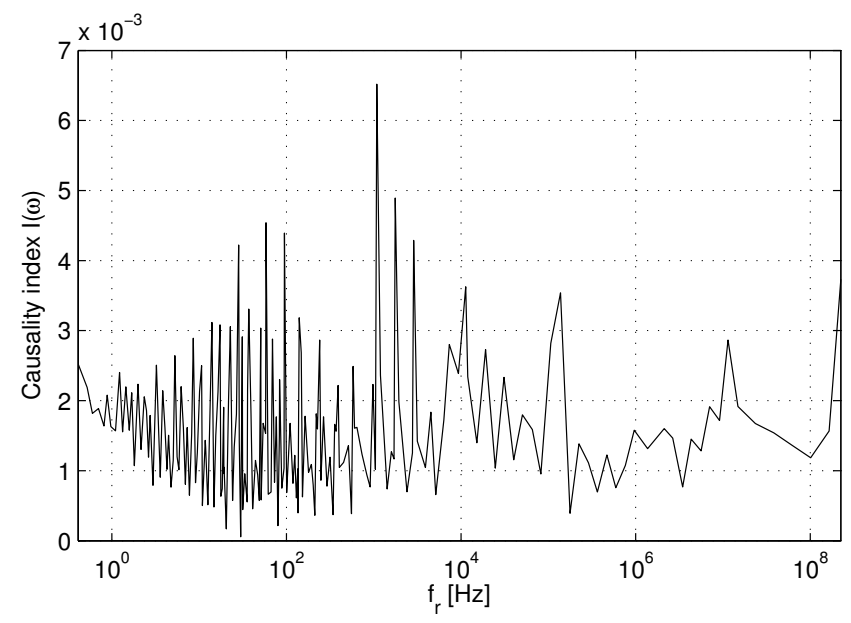

Figure 4. Causality index at the end of the optimization procedure, based on experimental measurements of Deltane 350.

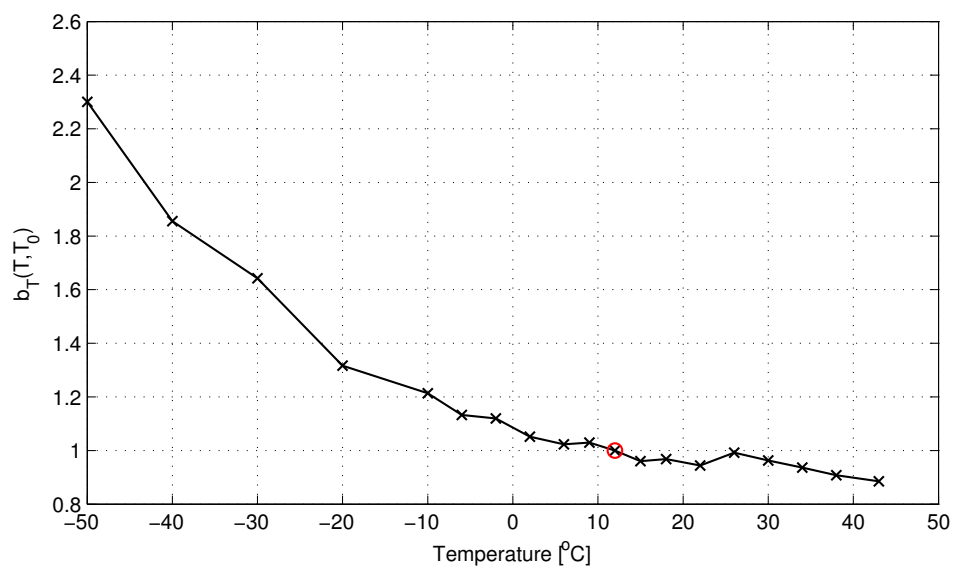

Figure 5. Temperature dependence of the function $b_{T}\left(T, T_{0}\right)$ used to obtain the master curves of the Deltane 350 , at a reference temperature of $T_{0}=12^{\circ} \mathrm{C}$. Crosses : temperature of measurements, circle : reference temperature. 

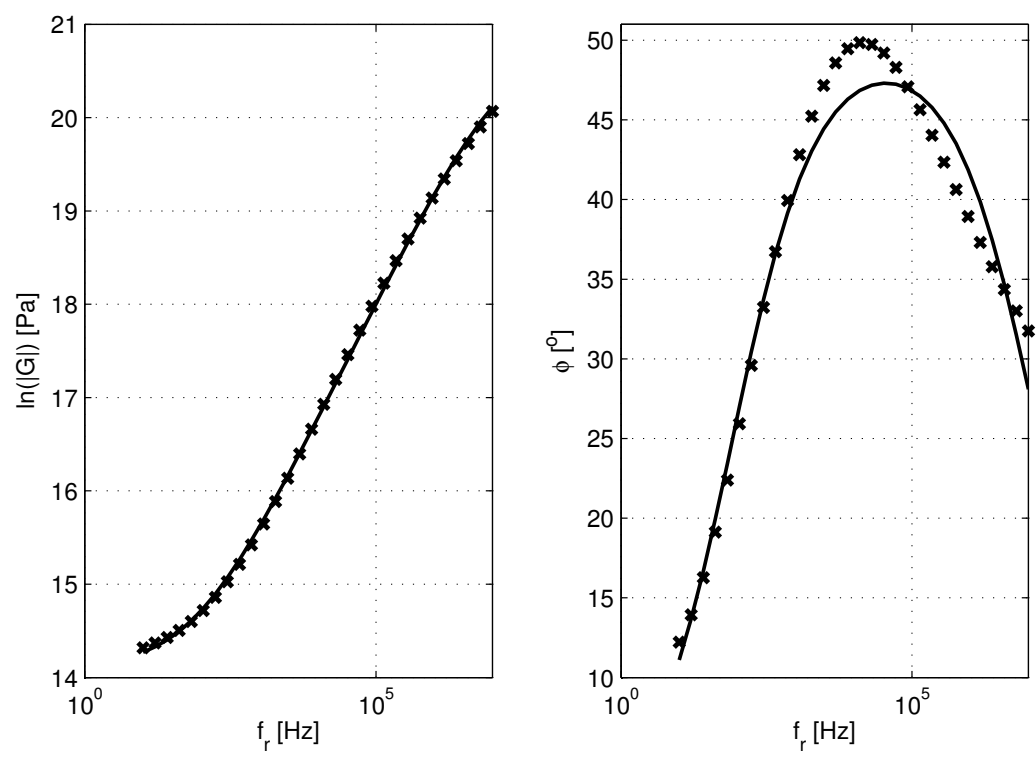

Figure 6. Master curves of Deltane 350 at a reference temperature of $12^{\circ} \mathrm{C}$.

Once the master curves are obtained, a viscoelastic model should be used in order to simulate the response of complex structures containing viscoelastic layers. In this work, a fractional derivative model is identified on the horizontally and vertically shifted data:

$$
G^{*}(\omega)=\frac{G_{0}+G_{\infty}(\mathbf{i} \tau \omega)^{\alpha}}{1+(\mathbf{i} \tau \omega)^{\alpha}}
$$

where $G_{0}=1.29 \mathrm{MPa}$ and $G_{\infty}=1.11 \mathrm{GPa}$ are the relaxed and unrelaxed modulus, $\tau=$ $1.0610^{-8} \mathrm{~s}$ is the relaxation time and $\alpha=0.56$ the order of the derivation. Figure 6 shows the master curves obtained with this model. The model is matching the modulus' amplitude but a discrepancy is observed on the phase angle. This can be improved by reducing the frequency range on which the curve fitting is performed or by enriching the viscoelastic model.

\section{REDUCED ORDER FINITE ELEMENT MODEL}

This section is devoted to the presentation of the reduced order model and its application to a case study: a bidimensional ring made of steel with partial viscoelastic core made of Deltane 350 and radial stiffeners made of steel. The structure, shown in Figure 7, is fixed at the intersection of the stiffeners and a unit load is applied at the left side.

The frequency dependence of the complex modulus of the viscoelastic material is described by the fractional derivative model of Eq.(9) identified on the master curves of Figure 6. The structure is meshed with 2-node beam elements with 4 degrees of freedom per node, as in [7] and [8]. This element is based on 'zigzag' theories : Timoshenko assumptions are made for the viscoelastic core, while Bernoulli assumptions are taken for the elastic faces. The system to be solved is :

$$
\left[\mathbf{K}_{e}+\frac{G^{*}(\omega)}{G_{0}} \mathbf{K}_{v}-\omega^{2} \mathbf{M}\right] \mathbf{u}=\mathbf{F}
$$




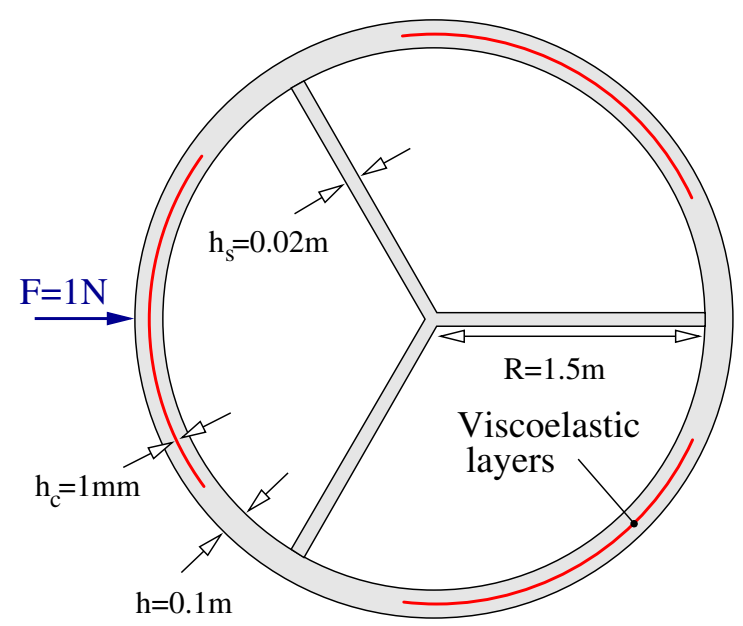

Figure 7. Description of the studied structure.

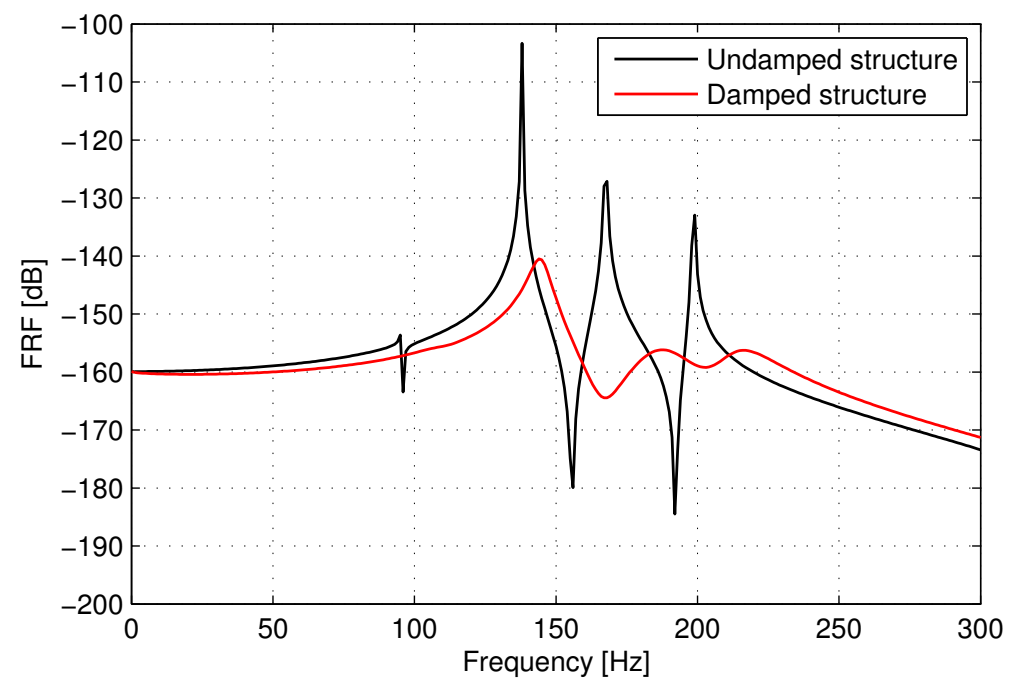

Figure 8. Reference frequency response of the undamped and damped structure calculated with a direct method.

where $\mathbf{K}_{e}$ is the stiffness matrix of the elastic faces, $\mathbf{K}_{v}$ is the stiffness matrix of the viscoelastic layer associated with the relaxed modulus $G_{0}, \mathbf{M}$ is the mass matrix of the whole structure, and $\mathbf{F}$ the load.

\subsection{Reference solution}

A direct method is used to calculate the reference frequency response of the structure with viscoelastic layer. It consists in solving Eq.(10) for each frequency. Figure 8 presents the frequency response of the undamped sandwich structure $\left(G=G_{0}\right)$ and the damped one $\left(G=G^{*}(\omega)\right)$. A significant reduction in the displacement levels is observed due to the frequency dependence of the mechanical properties of the viscoelastic material.

For more complex tridimensional models, the calculation of the frequency response using a direct method is time consuming and reduction methods are of great help. In view of structural and material optimization of the viscoelastic layer at the core of the ring, the 


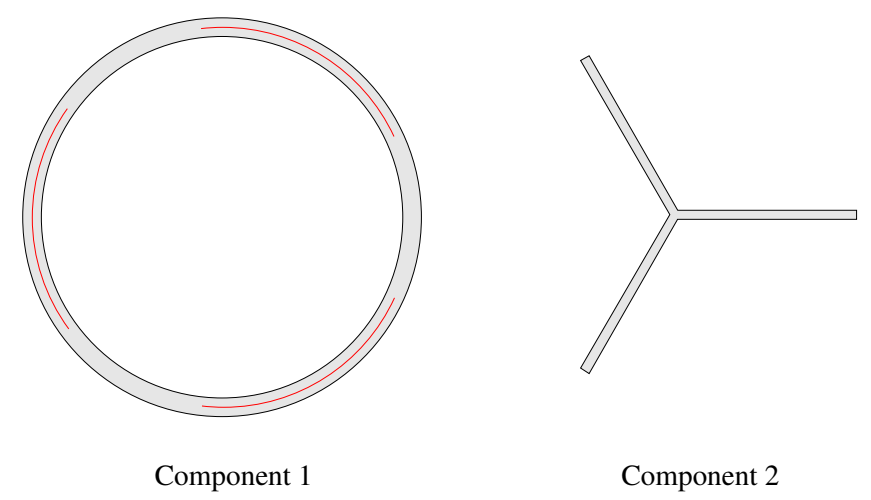

Figure 9. Substructuring of the structure of Figure 7.

dynamic substructuring approach presents some advantage. By considering the ring and the stiffeners as two components of the structure, it is possible to determine the new global behaviour of the structure induced by a local structural or material change in the viscoelastic layer only by recalculating the dynamic behaviour of the ring.

\subsection{Component mode synthesis}

Component synthesis method is a substructuring coupling method for which the structure is divided into components. For each component $i$, the degrees of freedom $\mathbf{u}^{i}$ are divided into interface degrees of freedom $\mathbf{u}_{\mathrm{J}}^{i}$ and internal degrees of freedom $\mathbf{u}_{\overline{\mathrm{J}}}^{i}$. The interface degrees of freedom are considered as unknowns and the internal degrees of freedom are condensed. By imposing continuity along interfaces, components are coupled and the dynamic behaviour of the overall structure can be calculated. The Craig-Bampton method is a reduction method commonly used to reduce the internal degrees of freedom in model substructuring. The CraigBampton reduction basis, in the first level of approximation, consists of low frequency fixedinterface normal modes $(\boldsymbol{\Phi})$ and a static condensation $\mathbf{T}_{\mathrm{s}}$ :

$$
\mathbf{T}_{\mathrm{CB}}=\left[\begin{array}{cc}
\mathbf{1} & \mathbf{0} \\
-\mathbf{K}_{\overline{\mathrm{J} J}}^{-1} \mathbf{K}_{\overline{\mathrm{J} J}} & \boldsymbol{\Phi}
\end{array}\right]
$$

where $\Phi$ contains real undamped fixed-interface modes $\phi_{i}$ of the component, solutions of:

$$
\left(\mathbf{K}_{e, \overline{\mathrm{J}} \overline{\mathrm{J}}}+\mathbf{K}_{v, \overline{\mathrm{J}} \overline{\mathrm{J}}}-\omega_{i}^{2} \mathbf{M}_{\overline{\mathrm{J}} \overline{\mathrm{J}}}\right) \boldsymbol{\phi}_{i}=\mathbf{0},
$$

and where

$$
\begin{aligned}
\mathbf{K}_{\overline{\mathrm{J}} \overline{\mathrm{J}}}=\left(\mathbf{K}_{e, \overline{\mathrm{J}} \overline{\mathrm{J}}}+\frac{\Re\left(G^{*}\left(\omega_{c}\right)\right)}{G_{0}} \mathbf{K}_{v, \overline{\mathrm{J}} \overline{\mathrm{J}}}\right) \\
\mathbf{K}_{\overline{\mathrm{J} J}}=\left(\mathbf{K}_{e, \overline{\mathrm{J} J}}+\frac{\Re\left(G^{*}\left(\omega_{c}\right)\right)}{G_{0}} \mathbf{K}_{v, \overline{\mathrm{J} J} \mathrm{~J}}\right)
\end{aligned}
$$

$\omega_{c}$ being the maximum frequency investigated. The structure represented on Figure 7 is divided into two components: the ring with viscoelastic layer, and the stiffeners.

The component mode synthesis is applied to the substructuring described on Figure 9. The first 48 real undamped modes are taken for each component. The frequency response 


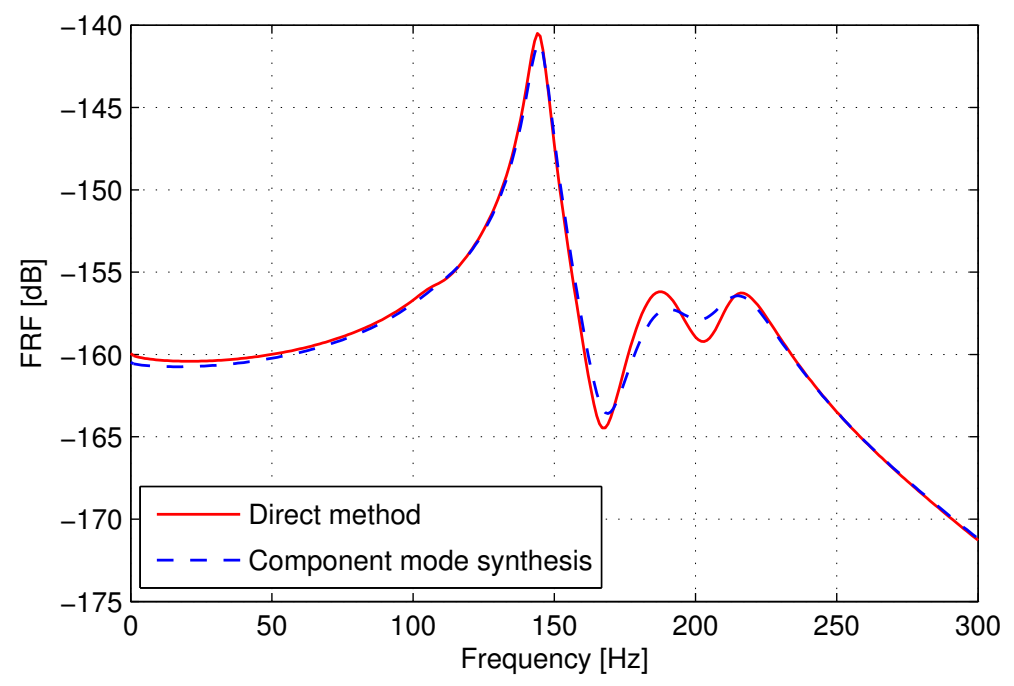

Figure 10. Frequency response of the structure using a direct method and a component mode synthesis method with 48 fixed-interface normal modes per component.

function of the structure calculated from this method is compared to the frequency responses obtained by a direct method on Figure 10. This method leads to errors in peak amplitudes and in damping. The real undamped modes are not sufficient to account for the frequency shift induced by the high frequency dependence of the viscoelastic properties.

In modal reduction techniques, where the same problem arises, a multi-model approach can be adopted to enrich the reduction basis and make it efficient for highly damped structures. It consists in adding a set of real normal modes associated with a high frequency modulus for the viscoelastic layer [9]. This same approach is now used to enrich the basis of fixed-interface modes in the Craig-Bampton basis.

\subsection{Enriched component mode synthesis}

The enriched Craig-Bampton reduction basis consists of fixed-interface normal modes associated with a real low frequency viscoelastic modulus $G=G_{0}$, fixed-interface normal modes associated with a real high frequency viscoelastic modulus $G=\Re\left(G^{*}\left(\omega_{c}\right)\right)$, and a static condensation :

$$
\mathbf{T}_{\mathrm{ECB}}=\left[\begin{array}{ccc}
1 & 0 & 0 \\
-\mathbf{K}_{\overline{\mathrm{J}} \overline{\mathrm{J}}}^{-1} \mathbf{K}_{\overline{\mathrm{J} J}} & \boldsymbol{\Phi} & \boldsymbol{\Psi}
\end{array}\right]
$$

where $\Psi$ contains the modes $\psi_{i}$, solutions of:

$$
\left(\mathbf{K}_{e, \overline{\mathrm{J}} \overline{\mathrm{J}}}+\frac{\Re\left(G^{*}\left(\omega_{c}\right)\right)}{G_{0}} \mathbf{K}_{v, \overline{\mathrm{J}} \overline{\mathrm{J}}}-\omega_{i}^{2} \mathbf{M}_{\overline{\mathrm{J}} \overline{\mathrm{J}}}\right) \boldsymbol{\psi}_{i}=\mathbf{0}
$$

This method is applied to the substructuring of Figure 9 with 24 normal modes associated with the viscoelastic relaxed modulus $G_{0}$ and 24 normal modes associated with a real viscoelastic modulus calculated at $300 \mathrm{~Hz}$, in addition to the static condensation. The results are fitting those obtained with a direct method, as shown on Figure 11. 


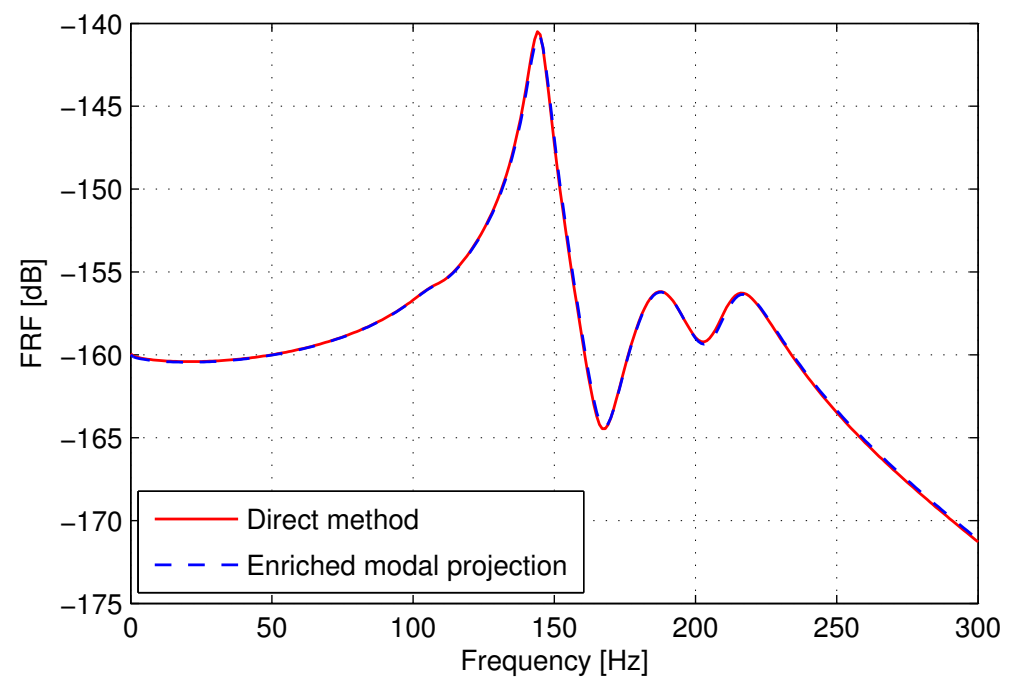

Figure 11. Frequency response of the structure using a direct method and an enriched component mode synthesis method with $2 \times 24$ fixed-interface normal modes per component.

\section{CONCLUSION}

In this work, a vertical shifting procedure is presented to build the master curves of imperfectly thermo-rheologically simple viscoelastic materials from dynamical mechanical analyzer measurements. This method is based on the exact Kramers-Kronig relationships that links the amplitude and the phase angle of the complex modulus. It imposes the master curves to fulfill the causality requirements. A fractional derivative model is then identified on the final master curves.

Another contribution of this work is to present a methodology to reduce the computational time to calculate the frequency response of a viscoelastically damped structure. A component modes synthesis method is tested on a viscoelastic sandwich structure. The case study shows that enriching the Craig-Bampton basis with real eigenmodes associated to a modulus calculated at a higher frequency gives better results than a standard Craig-Bampton basis at equal size of the reduction basis.

\section{ACKNOWLEDGMENTS}

The financial support of DGA/MRIS (Mission for Scientific Research and Innovation) and DCNS is gratefully acknowledged. 


\section{REFERENCES}

[1] Rouleau L., Deü J.-F., Legay A., Sigrist J.-F., "Vibro-acoustic study of a viscoelastic sandwich ring immersed into water", Journal of Sound and Vibration 331(3), 522-539, 2011

[2] Ferry J.D., "Viscoelastic properties of polymers", John Wiley New York, 1980

[3] Williams M.L., Landel R.F., Ferry J.D., "The temperature dependence of relaxation mechanisms in amorphous polymers and other glass forming liquids", Journal of the American Chemical Society 77(14), 3701-3707, 1955

[4] Fowler B., Rogers L., "A new approach to the vertical shift of complex modulus data for damping polymers", Proceedings of the 77th Shock and Vibration Symposium, Monterey, 2006

[5] Caracciolo R., Gasparetto A., Giovagnoni, "Application of causality check and of the reduced variable method for experimental determination of Young's modulus of a viscoelastic material", Mechanics of Materials 33(12), 693-703, 2001

[6] Parot J.-M., Duperray B., "Applications of exact causality relationships to materials dynamic analysis", Mechanics of Materials 39(5), 419-433, 2007

[7] Galucio A.C., Deü J.-F., Ohayon R., "Finite element formulation of viscoelastic sandwich beams using fractional derivative operators", Computational Mechanics 33(4), 282-291, 2004

[8] Trindade M.A., Benjeddou A., Ohayon R., "Finite element modelling of hybrid activepassive vibration damping of multilayer piezoelectric sandwich beams - Part I : Formulation”, International Journal for Numerical Methods in Engineering 51(7), 835-854, 2001

[9] Plouin A.-S., Balmès E., "Steel/viscoelastic/steel sandwich shells computational methods and experimental validations", Proceedings of the 28th International Modal Analysis Conference, San Antonio, 2000 